\title{
A Novel Control Strategy for Large-Capacity Energy Storage Systems Based on Virtual Synchronous Generator
}

\author{
Zhipeng $\mathrm{Wu}^{1,2, a, *}$, Ping Yang ${ }^{1, b}$, Dongyang Rui ${ }^{2}$ and Xinyu Jiang ${ }^{2}$ \\ ${ }^{1}$ School of Electric Power, South China University of Technology, Guangzhou 510641, China; \\ ${ }^{2}$ Guangzhou Zhiguang Electric Co., LTD, Guangzhou 510760, China. \\ awzp1986111@163.com, beppyang@gmail.com
}

\begin{abstract}
Large-capacity energy storage systems can meet the demands of micro-grid and the smart grid. But the traditional control method is difficult to realize plug and play and seamless switching between different operation modes, it has limited the roles of large-capacity energy storage system. Virtual synchronous generator (VSG) has became a hot topic in recent years because of its synchronous generator external characteristics, such as inertia, frequency voltage regulation and automatic phase lock. A novel control strategy based on VSG is proposed for large-capacity energy storage systems. Firstly, the topological structure characteristics of large-capacity energy storage systems and the basic principle of the novel control strategy based on VSG are introduced, then setting method of the control strategy parameters is given. Finally, the proposed control strategy is verified by simulation and experiment. The results of simulation and experiment show that large-capacity energy storage systems based on this novel control strategy can automatically adjust the active power output according to the grid frequency changes and realize seamless switching between connected-grid and off-grid mode.
\end{abstract}

Keywords: Virtual synchronous generator, energy storage systems, seamless switching, frequency and voltage regulation.

\section{Introduction}

With the rapid development of micro-grid and smart grid, the penetration rate of new energy such as wind power and photovoltaic (PV), which output is volatile and intermittent, is higher and higher in power grid. Energy storage system can improve the distributed power performance, ensure the reliability and quality of power supply and maintain the economic stability of power grid[1]. Therefore, energy storage technology research for the development and application of smart grid and micro-grid is of great significance.

Traditional energy storage systems usually adopt two-level or three-level circuit topology, but the single-machine capacity rarely achieves MW level, which greatly limits its roles of energy storage system. In recent years, large-capacity energy storage systems with cascade topology receive a lot of attention because of its topological structure characteristics[2, 3], the single-machine capacity can easily achieve MW even hundred MW level. However, the control methods of large-capacity energy storage systems are still traditional PQ and V/f control. Traditional control strategy cannot realize plug and play and real meaning seamless switching. Inspired by traditional synchronous generator, literature $[4,5,6]$ proposed virtual synchronous generator technologies or similar concepts. Through reasonable design, this control method can make the electronic power converters have the excellent characteristics of synchronous generator. And this method enables large-capacity energy storage system connect to the grid in a similar way of synchronous generator[7], expands the application range of large-capacity storage systems.

The topology structure of the large-capacity energy storage systems and the principle of the novel control strategy based on VSG are given in this paper firstly, then the setting method of this control 
strategy parameters is given. In this way, the large-capacity energy storage systems realize active power output regulation automatically and seamless switching between connected-grid and off-grid mode. Finally, simulation and experiment results demonstrate the feasibility and effectiveness of the proposed control strategy.

\section{Control Strategy Based on VSG for Large-Capacity Energy Storage Systems}

\subsection{Electrical Topology of Large-Capacity Energy Storage Systems.}

A 3-phase large-capacity energy storage system with $N$ power converter units per phase, which supplies electrical power to grid through inductors $\left(\mathrm{L}_{\mathrm{a}}, \mathrm{L}_{\mathrm{b}}\right.$ and $\left.\mathrm{L}_{\mathrm{c}}\right)$ is shown in Fig. 1. Here, each unit is a single-phase power converter which is electrically fed with an isolated battery pack. The addition of each unit output voltage in a phase produces the total output voltage[8, 9], which can reach 10KV even $35 \mathrm{KV}$ without transformer. The total capacity of this system is the sum of each battery pack capacity in three-phase, which can achieve MW even hundred MW level.

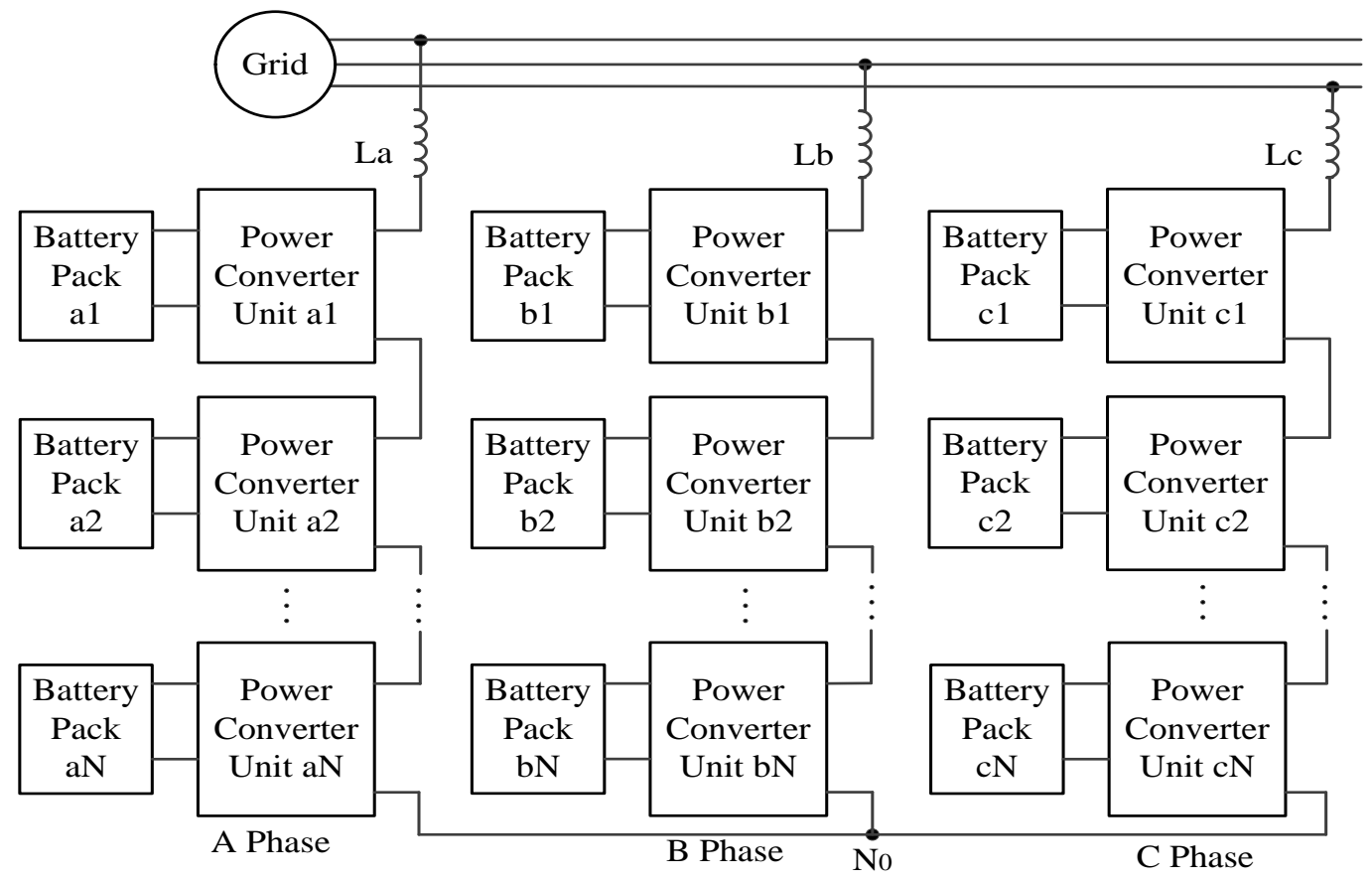

Fig. 1 The electrical topology of large-capacity energy storage systems

The electrical topology of each power converter unit is shown in Fig. 2.

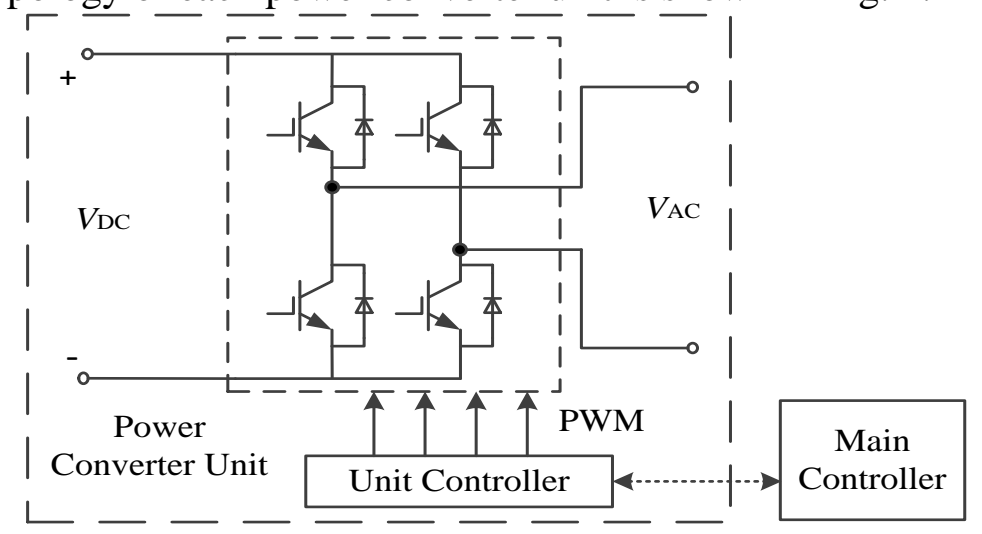

Fig. 2 The electrical topology of power converter units

As shown in Fig. 2, the core part of the power converter unit is a single phase H-bridge circuit [10], where $V_{\mathrm{DC}}$ is input of the H-bridge circuit, $V_{\mathrm{AC}}$ is output. Each power converter unit has an unit controller, which communicates with the main controller and performs control commands from the main controller. And the control algorithm based on VSG is running in the main controller. 


\subsection{Basic Principle of the VSG.}

VSG technology is put forward by the university of Leuven firstly [11]. Professor Qingchang Zhong perfected the VSG model, in addition to have frequency and voltage regulating function, but also reflect the electromagnetic transient process of synchronous generator [4]. The basic principle is as follows.

The induction electromotive force $e$ of synchronous generator can be represented as

$$
e=\left[\begin{array}{lll}
e_{a} & e_{b} & e_{c}
\end{array}\right]^{T}=M_{f} i_{f} \omega\left[\begin{array}{c}
\sin (\theta) \\
\sin (\theta-2 \pi / 3) \\
\sin (\theta+2 \pi / 3)
\end{array}\right]
$$

where $M_{f}$ is mutual inductance, $i_{f}$ is excitation current, $\omega$ is the angular frequency, $\theta$ is rotor angle.

Define the generated active power $P$ and reactive power $Q$ as

$$
\left\{\begin{array}{c}
P=\omega M_{f} i_{f}\left[\begin{array}{lll}
i_{a} & i_{b} & i_{c}
\end{array}\right] \cdot\left[\begin{array}{c}
\sin (\theta) \\
\sin (\theta-2 \pi / 3) \\
\sin (\theta+2 \pi / 3)
\end{array}\right] \\
Q=-\omega M_{f} i_{f}\left[\begin{array}{lll}
i_{a} & i_{b} & i_{c}
\end{array}\right] \cdot\left[\begin{array}{c}
\cos (\theta) \\
\cos (\theta-2 \pi / 3) \\
\cos (\theta+2 \pi / 3)
\end{array}\right]
\end{array}\right.
$$

where $i_{\mathrm{a}}, i_{\mathrm{b}}$ and $i_{\mathrm{c}}$ are output current in ABC three phase.

Thus the electromagnetic torque of synchronous generator can be represented as

$$
T_{e}=\frac{P}{\omega}=M_{f} i_{f}\left[\begin{array}{lll}
i_{a} & i_{b} & i_{c}
\end{array}\right] \cdot\left[\begin{array}{c}
\sin (\theta) \\
\sin (\theta-2 \pi / 3) \\
\sin (\theta+2 \pi / 3)
\end{array}\right]
$$

The mechanical equation of synchronous generator is given by

$$
J \dot{\omega}=T_{m}-T_{e}-D_{p} \omega
$$

where $J$ is the moment of inertia of all the parts rotating with the rotor, $T_{\mathrm{m}}$ is the mechanical torque, $T_{\mathrm{e}}$ is the electromagnetic torque, and $D_{\mathrm{p}}$ is a damping factor, which represents the mechanical-friction coefficient plus the frequency-drooping coefficient.

Thus the electromagnetic mechanical digital model of synchronous generator is shown in Fig. 3.

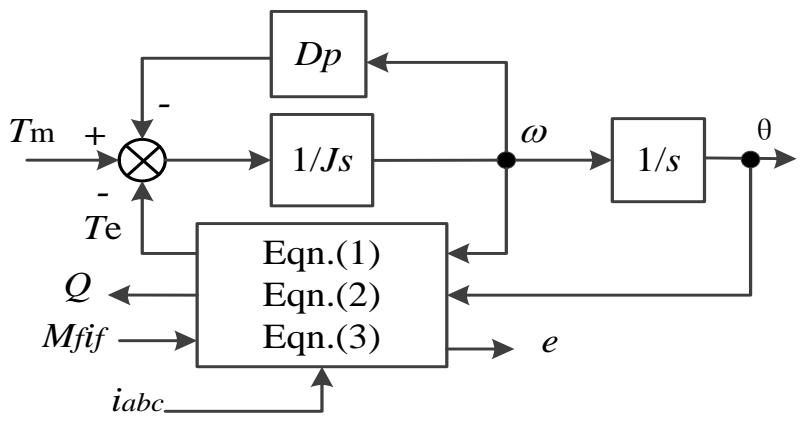

Fig. 3 The electromagnetic mechanical digital model of synchronous generator

This electromagnetic mechanical digital model is the core of VSG technology, which is implemented in digital signal processor (DSP). In this way, the power electronic converters have the similar external characteristic of synchronous generators.

\subsection{The Novel Control Strategy Based on VSG.}

Based on VSG, this paper proposes a novel control strategy for large-capacity energy storage systems, the control block diagram is shown in Fig. 4. As shown in this figure, $P_{\text {set }}$ and $Q_{\text {set }}$ are the given reference active and reactive power values, $\omega_{0}=2 \pi f_{0}\left(f_{0}=50 \mathrm{~Hz}\right)$ represents the fixed angular 
frequency, $P$ and $Q$ are actual measured values, $\Psi$ represents a integral gain, $D_{\mathrm{q}}$ is voltage-reactive power droop coefficient, $E_{0}$ is the fixed voltage amplitude, $E_{\text {out }}$ is the real output voltage amplitude, $E$ is the calculated reference voltage amplitude.

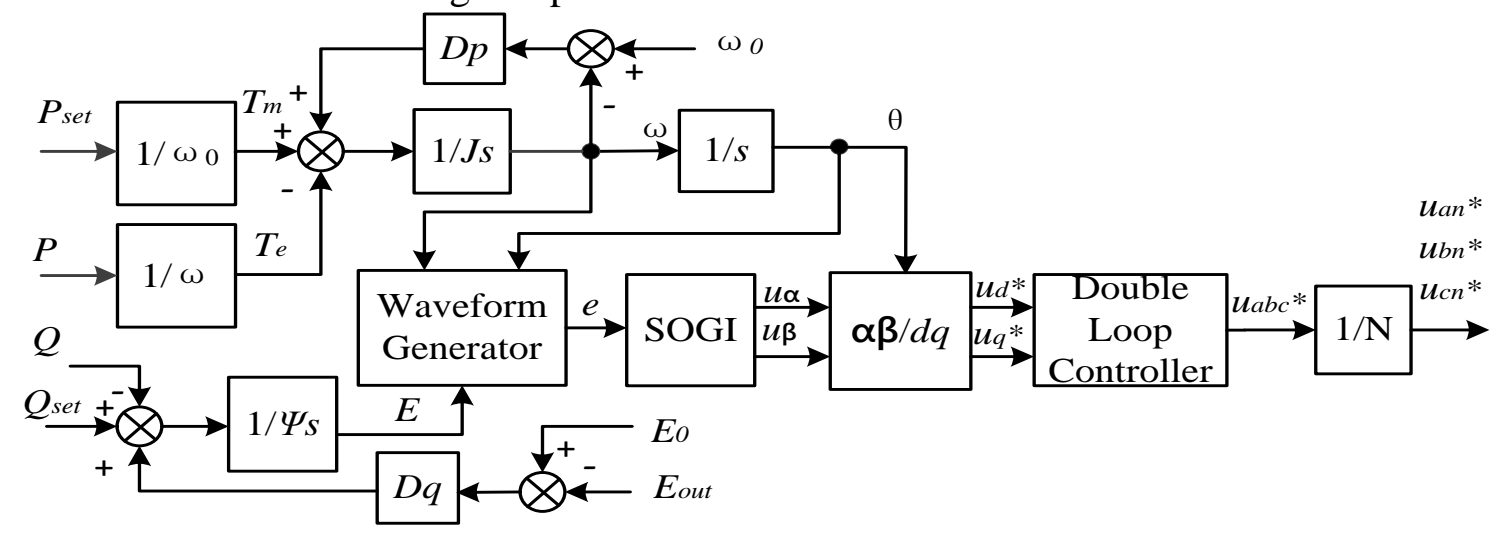

Fig. 4 Control block diagram of the novel control strategy based on VSG

Through the proposed control algorithm based on VSG, we get three elements of the reference voltage waveform, that is, amplitude $E$, frequency $\omega$ and phase $\theta$. Then the waveform generator generates reference voltage $e$. Because the reference voltage $e$ is a single-phase signal, therefore, the second-order generalized integrator (SOGI) is needed to get a pair of orthogonal signals $u_{\alpha}$ and $u_{\beta}$ [12]. After $\alpha \beta / \mathrm{dq}$ coordinate transformation, $u_{\mathrm{d}}{ }^{*}$ and $u_{\mathrm{q}}{ }^{*}$ are obtained. The three-phase reference voltage $u_{\mathrm{abc}}{ }^{*}$ of the large-capacity energy storage system are obtained by traditional voltage and current double loop controller, and then divided into $N$ equal parts ${u_{\mathrm{an}}}{ }^{*},{u_{\mathrm{bn}}}{ }^{*}$ and $u_{\mathrm{cn}}{ }^{*}(\mathrm{n}=1 \sim \mathrm{N})$, which are assigned to the unit controllers in each power conversion unit.

The setting principle of major parameters is given as follows

$$
\left\{\begin{array}{l}
D_{p}=-\frac{\Delta T}{\Delta \omega}=\left|\frac{\Delta P}{\omega \cdot \Delta \omega}\right|, D_{q}=-\frac{\Delta Q}{\Delta U} \\
J=D_{p} \cdot \tau_{f}, \Psi=\omega \cdot D_{q} \cdot \tau_{v}
\end{array}\right.
$$

where $\Delta T$ is the variation of electromagnetic torque (imaginary), $\Delta \omega$ is the allowed vary range of grid angular frequency, $\Delta P$ is the vary range of active power output, $\Delta Q$ is the vary range of reactive power output, $\Delta U$ is the allowed vary rang of grid voltage, $\tau_{f}$ is an inertial time constant, and $\tau_{v}$ is also a time constant, both of the two time constant should be selected small values.

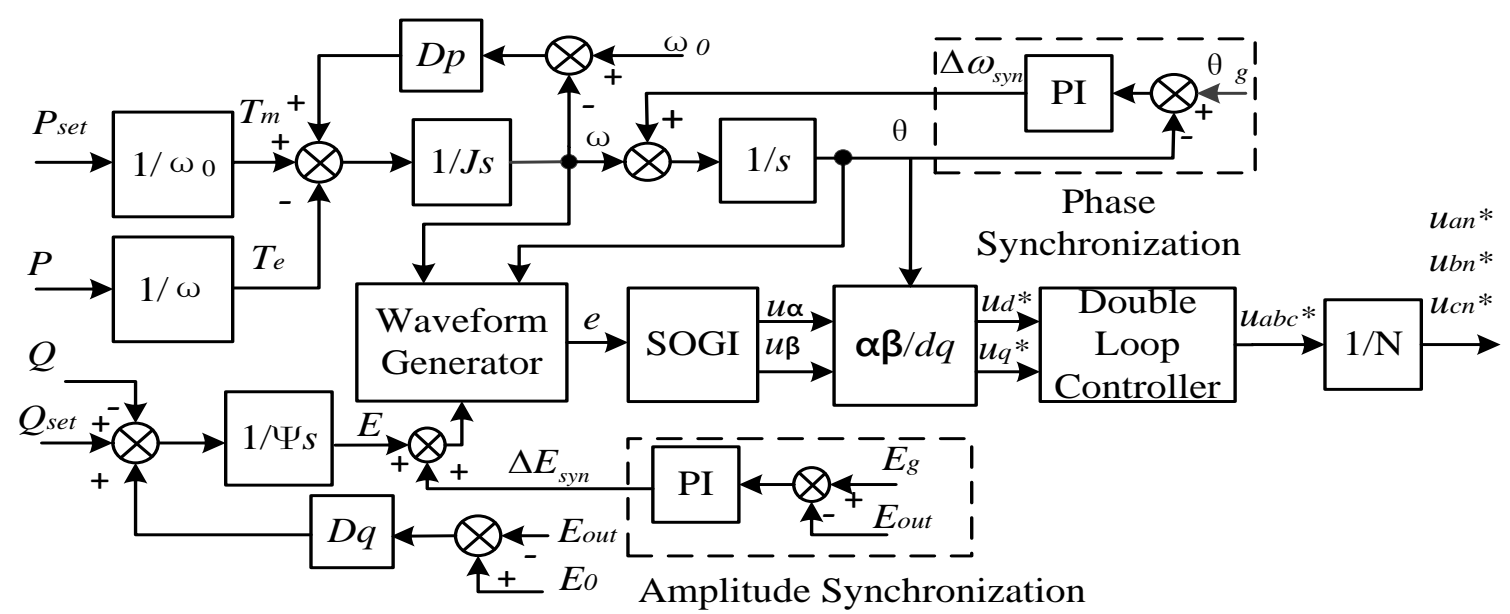

Fig. 5 Block diagram of synchronization control

The proposed control strategy makes large-capacity energy storage system always act as voltage-source model regardless of connected-grid or off-grid. Therefore, once the grid power-off, the proposed control strategy can automatically adjust output to match local load, there will be no over-voltage or over-current fault. However, when large-capacity energy storage system runs in off-grid model a period of time, the amplitude and phase of its output voltage may be different with 
grid voltage. There will be large impulse current, if system connects to the grid directly in this case. In order to realize seamless switching, the proposed control strategy designs presynchronization control, as shown the dashed areas in Fig. 5. In this figure, $\theta_{\mathrm{g}}$ is the phase of grid voltage obtained by PLL, $E_{\mathrm{g}}$ is the measured amplitude of grid voltage, $\Delta \omega_{\text {syn }}$ is the compensation of phase deviation, and $\Delta E_{\text {syn }}$ is the compensation of amplitude deviation. When receives the connecting grid command, the system cut-in the presynchronous control until the phase and amplitude are synchronous with grid voltage completely.

\section{Simulation and Experiment Results}

Under the MATLAB/Simulink, this paper set up simulation system to demonstrate the effectiveness of the proposed control strategy. Experiments based on hardware platform with the same parameters were carried out to test and verify this control strategy also, and a programmable power supply was used to replace the grid. The parameters of the system used in the simulation are given in Table I.

The simulations and experiments can be divided into two major parts. The first one is the active power-frequency droop characteristic validation, the second one is demonstrating the seamless switching between connected-grid and off-grid mode.

Table 1 Parameters of the simulation system

\begin{tabular}{|c|c|c|c|}
\hline Parameters & Values & Parameters & Values \\
\hline rated voltage & $10 \mathrm{KV}$ & converter units number & $20 /$ phase \\
\hline rated power & $2 \mathrm{MW}$ & $L_{\mathrm{a}}, L_{\mathrm{b}}, L_{\mathrm{c}}$ & $2 \mathrm{mH}$ \\
\hline$f_{0}$ & $50 \mathrm{~Hz}$ & $D_{\mathrm{p}}$ & 1267 \\
\hline DC voltage & $720 \mathrm{~V}$ & $D_{\mathrm{q}}$ & 7885 \\
\hline$\tau_{\mathrm{v}}$ & $0.004 \mathrm{~s}$ & $\tau_{\mathrm{f}}$ & $0.004 \mathrm{~s}$ \\
\hline
\end{tabular}

Fig. 6 is the demonstration of active power-frequency droop characteristic, the given power output of this simulation system was $1 \mathrm{MW}$, normal frequency was $50 \mathrm{~Hz}$, and decreased to $49.5 \mathrm{~Hz}$ at $0.2 \mathrm{~s}$, raised to $50.2 \mathrm{~Hz}$ at $0.35 \mathrm{~s}$. As shown in this figure, this system adjust active power output following the frequency changes automatically. The experiment results also confirmed this feature.
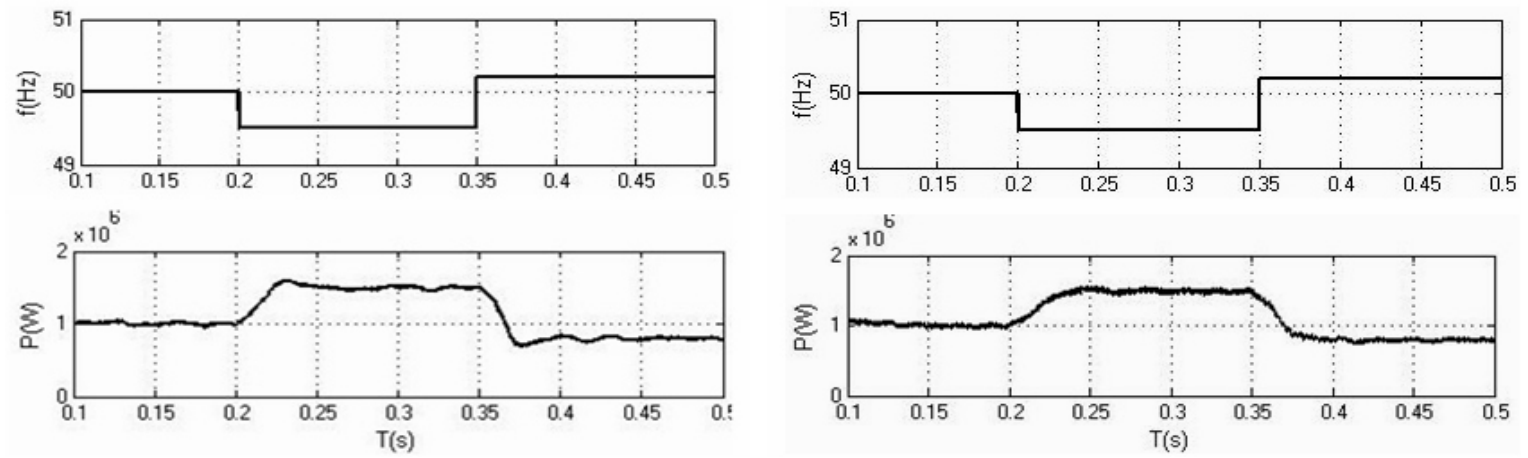

Fig. 6 Waveforms of active power-frequency droop, left is simulation result, right is experiment

Fig.7 shows the seamless switching of this proposed control strategy, the grid power-off at $0.25 \mathrm{~s}$ and restore power supply at $0.35 \mathrm{~s}$, when the grid power-off, the energy storage system supply power to the local load. As shown in this figure, the upper waveform is the output current of this system, the 

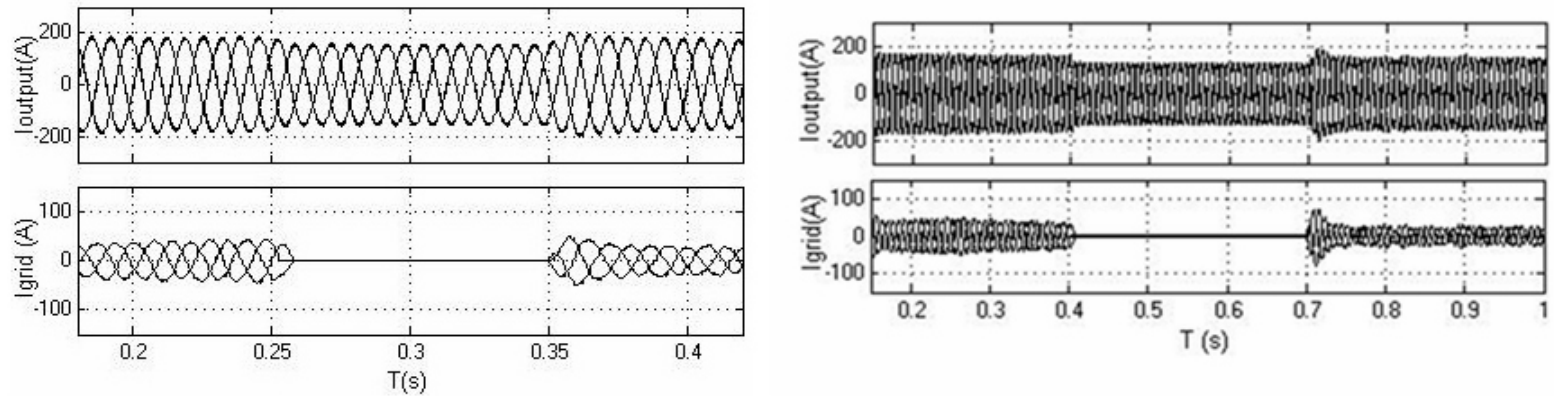

Fig. 7 Seamless switching demonstration, left is simulation result, right is experiment result lower one is current fed into grid. There is no obvious impact current regardless of off-grid or connected-grid. In the experiment, a solid-state switch was used to simulate grid power-off and interconnection to the grid, which was off at $0.4 \mathrm{~s}$ and on at $0.7 \mathrm{~s}$. Experiment results show no current peak regardless of off-grid or connected-grid also.

\section{Conclusions}

In this paper, the idea of operating large-capacity energy storage systems as SGs has been proposed and proved to be feasible after establishing a control strategy based on VSG. The implementation and operation of such an large-capacity energy storage system, including power regulation and seamless switching, have been developed and described in detail. Both simulation and experimental results are provided.

\section{References}

[1] G. Li, Y. Tao, J. Hu and G. Su, Research on control strategy of energy storage system in microgrid, Power Electronics, 47 (2013) 9-11.

[2] Q. Miao, J.Y. Wu and H. Ai, Coordinated contro1 of hybrid cascaded megawatt power regu1ation device, E1ectric Power Automation Equipment, 34 ( 2014) 43-49.

[3] L. Maharjan, S. Inoue and H. Akagi, A transformer1ess energy storage system based on a cascade mu1ti1eve1 PWM converter with star configuration, IEEE Transactions on Industry App1ications, 44 (2008) 1621-1630.

[4] Q.C. Zhong and G. Weiss, Synchronverters: inverters that mimic synchronous generators, IEEE Transactions on Industrial Electronics, 58 (2011) 1259-1267.

[5] T. Shintai, Y. Miura and T. Ise, Oscillation damping of a distributed generator using a virtual synchronous generator, IEEE Transactions on Power Delivery, 29 (2014) 668-676.

[6] Y. Chen, R. Hesse and D. Turschner, Comparison of methods for implementing virtual synchronous machine on inverters, 2012 International Conference on Renewable Energies and Power Quality, 2012, Santiago, Spain.

[7] N. Tang, C. Sheng and M. Chen, Review on virtual synchronous generator technologies, Southern Power System Technology, 10 (2016) 30-38.

[8] R. Baidya, R. Aguilera, P. Acuna, S. Vazquez and H.D. Mouton, Multistep model predictive control for cascaded H-bridge inverters: formulation and analysis, IEEE Transactions on Power Electronics, pp (2017) 1-1.

[9] B. Wu and M. Narimani, Cascaded H-bridge multilevel inverters, High-Power Converters and AC Drives, Second Edition, Wiley-IEEE Press, Piscataway, 2017, pp. 119-141.

[10] R. Pandey, R.N. Tripathi and T. Hanamoto, Cascaded H-bridge multilevel inverter for renewable energy generation, Journal of Physics: Conference Series, 704 (2016) 12-20. 
[11] K. Visscher and S.W.H. De Haan, Virtual synchronous machines (VSG's) for frequency stabilisation in future grids with a significant share of decentralized generation, Smartgrids for Distribution, Iet-cired Cired Seminar, 2008:1-4.

[12] M. Ciobotaru, R. Teodorescu and F. Blaabjerg, A new single-phase PLL structure based on second order generalized integrator, 37th IEEE Power Electronics Specialists Conference, 2006, Jeju, Korea. 